\title{
Joint control actions on electromechanical devices in ergatic systems
}

\author{
Alexandr Saushev ${ }^{1, *}$, Vladimir Tyrva ${ }^{1}$, and Lev Kovtun ${ }^{1}$ \\ ${ }^{1}$ Admiral Makarov State University of Maritime and Inland Shipping, Dvinskaya Str., 5/7, \\ Saint Petersburg, 198035, Russia
}

\begin{abstract}
The problem of implementing joint control actions on electromechanical devices (EMD) in human-machine systems through the control body of a human-machine interface is considered. Based on the theory of optimal control, control functions between the human operator, the program control automaton, and the corrective control automaton are synthesized. Peculiarities of the tech-nical implementation of the control actions on the EMD of the ergatic system using the new type of joint control devices have been identified, through the control body of which information interaction between the human operator and the system automa-ton is carried out. Based on the model of actions and responses of the human operator and machine, which is adopted in engi-neering psychology, mathematical models of their joint control of the object have been developed. Mathematical models of ma-chine responses are constructed using the hypothesis of complete certainty in the form of a normal system of ordinary differen-tial equations. As a result of combining action and response models for the human-machine system, a finite set of incomplete representations of the elementary motions of the image point in the state space is obtained, on which the programmed motion of the object is built in the form of a sequence of elementary motions. Virtual discrete control signals corresponding to specific positions of the control bodies of the joint control apparatuses of the human-machine interface of the system are determined, through which consecutive transitions from one elementary movement to another are carried out. The conclusions on the effec-tiveness of the use of joint control in transport ergatic humanmachine systems are formulated. It is shown that in this case, the advantages of control partners are used and combined to the greatest extent.
\end{abstract}

\section{Introduction}

The problem of the distribution of control functions between a human operator and a machine in an ergatic system does not lose its relevance when creating new automated systems $[1,2]$. The main requirement for the implementation of the joint impact on the controlled object, for which the EMD is considered, is to improve the quality of control in comparison with only manual control. Manual control can come from a human operator or

\footnotetext{
* Corresponding author: $\underline{\text { saushev@bk.ru }}$
} 
a control automaton [3-5]. If we take into account that manual control in the ergatic human-machine system is carried out using the control unit of the human-machine interface of the system, then it is relevant to study the possibilities of controlling the object of the ergatic system through this control unit, simultaneously from the side of the human operator and the control automaton of the system when combining control actions. The technical implementation of joint control of an object should correspond to such an algorithmization of the actions of a human operator and a control automaton, in which control according to the selected criterion would be optimal [3].

To date, there are no theoretical and experimental studies and models that fully support the use of combined control by "control partners" in the design of ergatic human-machine systems. In this regard, the task of studying the capabilities and features of the implementation of the combined control actions on the EMD in the human-machine systems through the control device of the human-machine interface is considered.

\section{Materials and methods}

\subsection{Options for the separation and combination of control functions in the human-machine system}

The synthesis of possible options for the distribution of control functions in the humanmachine system is based on the hypotheses of "separation", "complete certainty" and "linearization" used in the theory of optimal control. The first hypothesis allows considering the object control problem in the form of two components: the program motion problem and the program motion control problem in a certain state space. In accordance with these problems, two stages of designing the control process are distinguished, which can be formalized and allow obtaining optimal and technically feasible solutions. The first stage involves the construction of a programmed trajectory of the object using the hypothesis of complete certainty. The second stage involves the synthesis of the control process, i.e. obtaining such control that should ensure the implementation of the program under the conditions of perturbations. In solving the synthesis problem, the linearization hypothesis is usually used. To solve these problems, each of them can be matched with its "own" automaton as part of the control automaton of the ergatic system. For the first problem, this is a program control automaton. For the second problem, this is a corrective control automaton for deviations from the programmed motion of the EMD.

Possible options for distributing the object control functions between the human operator (HO), the program control automaton (PCA) and the corrective control automaton (CCA) are given in Table 1.

Table 1. Distribution of control functions between a human operator and automatons of the human-machine system

\begin{tabular}{|c|c|c|c|c|}
\hline & \multicolumn{3}{|c|}{$\begin{array}{l}\text { Implementation of control } \\
\text { functions }\end{array}$} & \multirow[t]{2}{*}{ Characteristic of the implemented control } \\
\hline & PCA & $\mathrm{CCA}$ & $\mathrm{HO}$ & \\
\hline 000 & no & no & no & The human-machine system is idle \\
\hline 001 & no & no & yes & Manual (remote) control of an object \\
\hline 010 & no & yes & no & $\begin{array}{l}\text { Automatic control by set-point (automatic } \\
\text { motion stabilization) }\end{array}$ \\
\hline 011 & no & yes & yes & $\begin{array}{l}\text { Joint control (HO+CCA) - HO sets the set-point } \\
\text { for CCA (automatic tracking) }\end{array}$ \\
\hline 100 & yes & no & no & Automatic open loop control \\
\hline 101 & yes & no & yes & 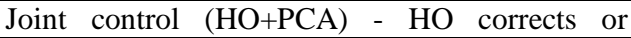 \\
\hline
\end{tabular}




\begin{tabular}{|c|c|c|c|l|}
\hline 110 & yes & yes & no & $\begin{array}{l}\text { changes the program } \\
\text { controlled movement, or/and trains automatons, } \\
\text { or is not in the control system of the object }\end{array}$ \\
\hline 111 & yes & yes & yes & $\begin{array}{l}\text { Joint control (HO+PCA+CCA) - HO controls the } \\
\text { object, and the automatons control the object at } \\
\text { the same time as HO }\end{array}$ \\
\hline
\end{tabular}

Examples of control systems are known for each variant of the distribution of control functions from the table. However, they do not use such joint control apparatuses in which control actions on the object from the side of the human operator and automatons of the ergatic system would be combined through the control device of the apparatus.

\subsection{Analysis of the features of the technical implementation of joint control}

In a formal presentation, the joint action on the object from the side of the human operator and the control automaton of the human-machine system occupies an intermediate position between manual control and automatic control of the object, i.e. it is a kind of automated control.

Manual control is implemented using the controls of electrical and electronic devices of the human-machine interface of the ergatic system. Manual control devices (buttons, breakers, switches, command controllers, rheostats, joysticks, etc.) are used, which perceive the muscular effort of a human operator applied to the control unit of the apparatus and respond to it by moving the control body. This movement of the control body is interpreted by the human operator as a control signal for the object to change its state, transmitted to the system using the manual control device. Therefore, if a human operator can observe the movement of the control body when it is performed by the controlling automaton, he will present this action as the "intention" of the automaton to change the state of the object in a certain way with such a signal.

In automatic control of an object, an observer (let's call it a human operator) can perceive a change in the position of the control body as a visual signal and as a tactile signal if the hand holds the control body and moves with it. The human operator cannot independently change the position of the control body when the object is automatically controlled. In this case, the control system of the object, in essence, is not an ergatic system, but will "come close" to that if it provides for the ability to change the position of the control body created by the control automaton with the help of a human effort. The participation of the human operator in the joint control of the object allows considering this system as an ergatic human-machine system. In such systems, information on the intent of the control automaton can be received by a human operator, along with visual signal, with two components of the tactile signal - the movement of the control body and the force of the control body on the arm.

The use of the joint control apparatus makes it possible to implement, in the process of controlling an object at separate time intervals, all the options for distributing control functions between a human operator and automatons of the human-machine system, presented in Table 1. Information links between the components of the human-machine system, which are important when using the joint control apparatus (JCA), are presented in Fig. 1. 


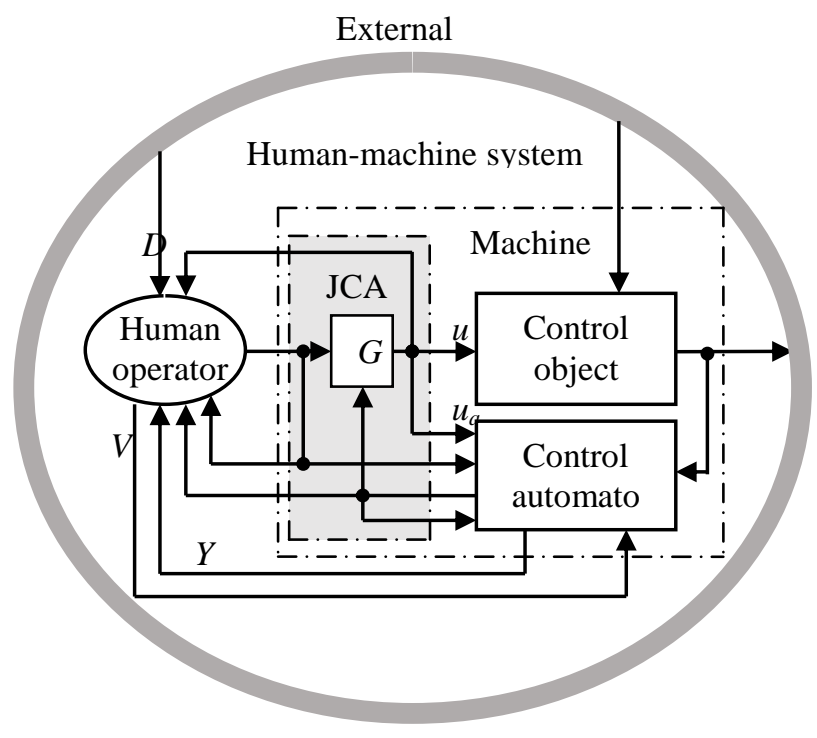

Fig. 1. The functional structure of the human-machine system with the JCA apparatus for the joint control implementation

Figure 1 shows that the control of an object is carried out using JCA under conditions when perturbations $S$ act on the object and the human operator has information links $D$ and $V$ - with the external environment and with the control automaton, respectively. Joint (resulting) control $u$ of an object is the result of a transformation of the control action $u_{c}$ created by the human operator and the control action $u_{a}$ coming from the control automaton. Wherein:

$$
u=G\left(u_{c}, u_{a}\right),
$$

where $G$ - conversion operator, which may change during the process of controlling the object. The result of control $u$ is the change in time $t$ of the state parameters (phase coordinates) $X$ of the object, information $Y$ about which, according to the measurement results, is transmitted to the human operator using the human-machine interface.

When the control actions on the object are combined in time, the control system of the JCA of the human-machine interface should be able to move both under the muscular effort of the human operator and under the action of the command generated by the control automaton of the human-machine system. In the joint control apparatus, a drive and converter devices must be provided. The first of them drives the control body on command from the control automaton, while maintaining the ability to move the control body with the muscular effort of the human operator, and the second generates a control signal in the form of a functional dependence on the combined control actions, i.e. into the signal to which the EMD responds by changing its state [6].

\subsection{Models and implementations of joint control through discrete devices}

For discrete devices, including contact devices, the following mathematical models of the positions of the control body during manual control (actions of the human operator) are used [6, 7]:

- two-position control model (2P model): 


$$
\left.\begin{array}{l}
u=u_{O}-" o n " \\
u=0-" o f f "
\end{array}\right\}
$$

- three-position control model (3P model):

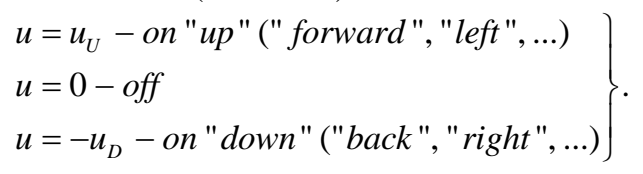

The parameters $u_{O}, u_{U}, u_{D}$ are given some positive values.

The basis for the construction of a joint control apparatus is the integration of a mechanical manual control drive with an electromechanical drive remotely controlled from an automaton of the ergatic system. The number of possible designs is not limited. An example of the implementation of an apparatus for joint control with a polarized electromagnetic drive is shown in Fig. 2, a.

a)

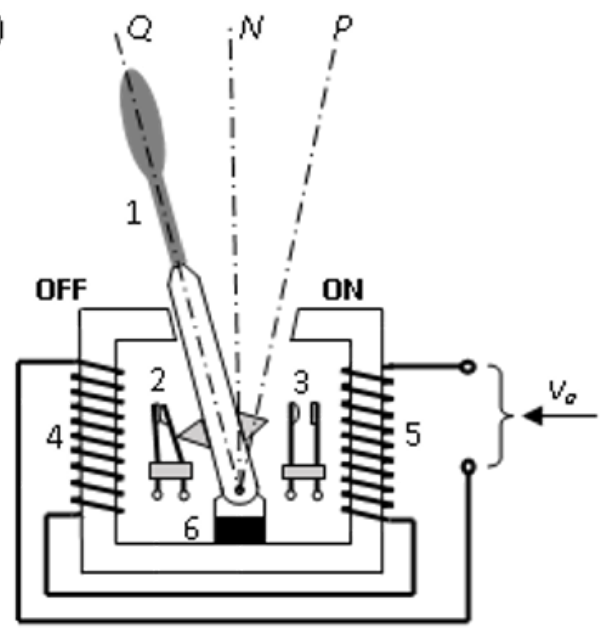

b)

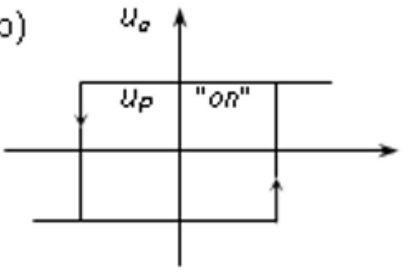

c)

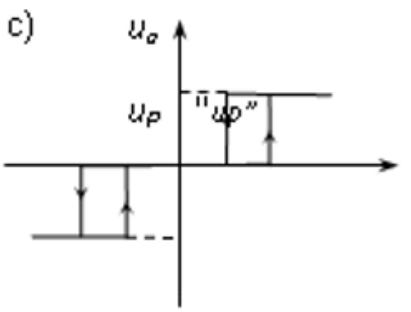

Fig. 2. A graphic model of a joint control apparatus intended for the implementation of $2 \mathrm{P}$ and $3 \mathrm{P}$ models (a) and control characteristics of the apparatus during the implementation of $2 \mathrm{P}(\mathrm{b})$ and $3 \mathrm{P}(\mathrm{c})$ models

For a human operator, the signal $v_{a}$ (electric voltage) applied to the windings 4,5 of a polarized electromagnetic drive with a permanent magnet 6 as part of the magnetic circuit (Fig. 2,a) is the intention of the control automaton to set the control body 1 to a certain position $Q$ or $P$ (depending on the polarity of the signal $v_{a}$ ). If this happens, then an object control signal $u=u_{a}$ is formed (Fig. 1), which is transmitted to the controlled object for execution by means of a closing contact, for example, contact 3 (Fig. 2). If the human operator, holding the handle 1 of the device, prevents its movement, despite the presence of the signal $v_{a}$, then the result will be the signal $u=u_{c}$ from the human operator, despite the action of the control automaton, which remains only an "intention", since it is not executed by the joint control apparatus (Fig. 1). 2P model (2) of the two-position control $(Q$ and $P$ ) corresponds to the control characteristic of the device, shown in Fig. 2, b. The signal $u_{a}=u_{0}$ corresponds to the coordinate $u_{P}$, the signal $u_{a}=0$ corresponds to the coordinate $u_{Q}$.

The operation of the apparatus (Fig. 2, a) can be described by 3P model (3) if its drive is supplemented with a return spring, which transfers the control body 1 to the neutral position $N$, when the signal $v_{a}=0$, and both contacts 2 and 3 are used to form an impact on the controlled object by position $u$ of the control body. The action of the apparatus by signals from the control automaton in this case is determined by the control characteristic shown in 
Fig. 2, c. The design parameters of the apparatus can be selected so that the human operator can impede the movement of the control body by a signal from the control automaton.

When performing joint control, the human operator must control the position of the control body corresponding to the signal $u$ (Fig. 1). In the example with the apparatus shown in Fig. 2,a, the intent of the control automaton is transmitted to the hand of the human operator in the form of a tactile signal $\left(u_{a}\right.$ in Fig. 1$)$ by force from the control body. Joint control apparatuses with various combinations of visual, sound and tactile signals are possible when transmitting information to a human operator about the intentions of a control automaton.

From the principle of the apparatus operation (Fig. 2) it follows that the priority in controlling the object is given to the human operator in comparison with the control automaton. If the human operator agrees with the intention of the automaton and does not impede the movement of the control body, the object is essentially controlled by the automaton. In case of disagreement of actions (intentions) - the human operator takes control of the object, despite the intentions of the automaton. To implement joint control, it is necessary for the human operator to control the position of the control body corresponding to the signal $u$ of controlling the object and the intention $u_{a}$ of the control automaton (Fig. 1).

\subsection{Models and implementations of joint control based on continuous apparatus}

For continuous apparatuses, the following mathematical models of the positions of the control body during manual control (actions of the human operator) in which the human operator can manifest itself as a source of speed or as a source of force have been proposed $[6,7]$ :

- multi-position control model (MP model):

$$
\dot{u}=\omega,
$$

$\omega=\omega_{\mathrm{U}}$ - change "up" the position of $\mathrm{CB}$

$\omega=0-$ do not change the position of $\mathrm{CB}\}$;

$\omega=-\omega_{D}$ - change "down" the position of CB

- model of a multi-position multi-speed control element (MPS model):

$$
\left.\begin{array}{l}
\dot{u}=\omega \\
\dot{\omega}=\varepsilon
\end{array}\right\},
$$

$$
\left.\begin{array}{l}
\varepsilon=\varepsilon_{\mathrm{U}} \text { - change "up" the speed of CB movement } \\
\varepsilon=0 \text { - do not change the speed of CB movement } \\
\varepsilon=-\varepsilon_{\mathrm{D}} \text { - change "down" the speed of CB movement }
\end{array}\right\} \text {. }
$$

The parameters $\omega_{\mathrm{U}}, \omega_{\mathrm{D}}, \varepsilon_{U}, \varepsilon_{\mathrm{D}}$ are given some numerical positive values. The dot over the letter indicates the operation of differentiation with respect to time $t$.

Joint control apparatuses, as analogues of the corresponding continuous control apparatuses of continuous manual operation, must be implemented in such a way that the movements $u_{a}$ of the apparatus control body by signals from the control automaton are carried out similarly to the manual control apparatuses and are modeled by the same expressions (4), (5) or (6), (7). The basis for the construction of a joint control apparatus can be based on the integration of a manual mechanical drive with an electromechanical drive, for example, with a positional electric drive remotely controlled from an automaton of an ergatic system [5]. The number of possible options for the technical implementation of the apparatus is not limited. 
Fig. 3, a reveals the principle of constructing a joint control apparatus with a multiposition control body 1 and an analog sensor of angular displacement 3 . The signal $v$ at the output of the sensor is uniquely determined by the position $u=u_{a}+u_{c}$ of the manual control handle (position $Q$ ). By means of springs, the control handle 1 is connected to a steering head 2, which is deviated from the neutral position $N$ to a variable position $P$ by an electromechanical transducer 5 with a worm gear 4 . The angular velocity of the structural elements of the apparatus: $\omega_{n}$ - mechanical transmission of the worm $4, \omega_{a}$ - head $2, \omega_{c}$ handle 1 relative to the head 2 .

The angle of rotation $u_{a}$ is set by the control automaton of the human-machine system by the signal $v_{a}$. For example, in an airplane, such a movement of the control handle is carried out using ACS (automatic control system) with $u=u_{a}$ [8-10]. In aviation, the transmission of information about the intent of ACS was verified using a visual signal (in Fig. 3, a, by the position of the arrow) and using a tactile signal (in Fig. 3,a, by the force of counteraction from the side of the springs to the muscular effort of the human operator). HOTAS control method (Hands On Throttle-And-Stick) received a positive assessment. The difference from the control considered in Fig. 3, a consists in the use of an additional signal, according to which the transition from manual control to automatic control from ACS and in the reverse order is carried out.

a)

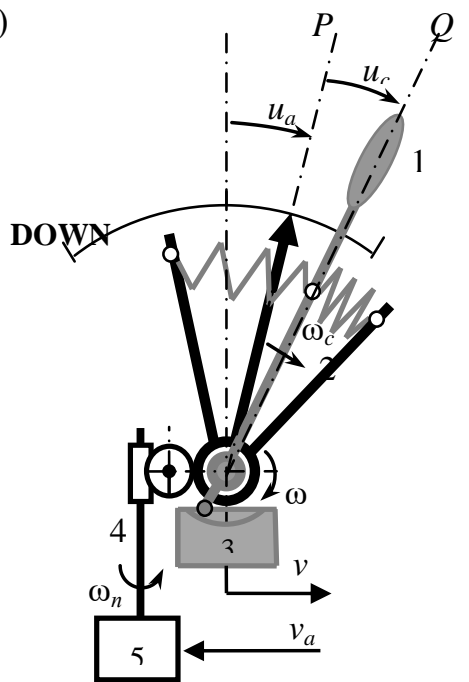

b)

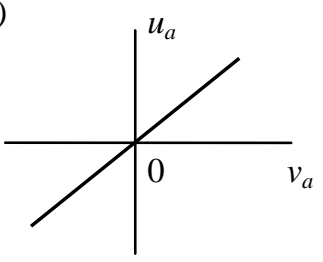

c)

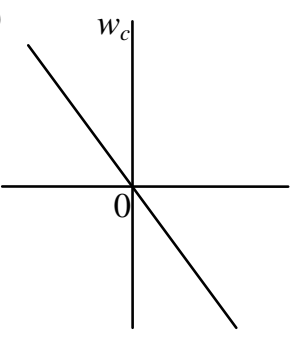

Fig. 3. A graphic model of the joint control apparatus intended for the implementation of the MP and MPS models (a), the control characteristic of the apparatus (b), the dependence of the response of the control body to its movement by muscular effort (c)

Figure 3,b shows the control characteristic of the apparatus in the linear version of the static dependence of $u_{a}$ on $v_{a}$. Thanks to the worm gear 4 , the connection between the electromechanical converter 5 and the head 2 is unidirectional - from the converter to the head. Therefore, the control actions of a human operator when moving a control body do not affect the operation of the control automaton of the human-machine system and do not change its signal $u_{a}$, implemented in the apparatus in the form of a certain position of the control body. When the human operator holds the control body with his hand, then through the movement of the handle and the forces that have arisen $\left(w_{c}\right.$ in Fig. 3,c) due to the elasticity of the springs, he receives information about the intentions of the control automaton to change the current state of the object. In this case, the human operator, by moving the control body (to position $Q$ in Fig. 3,a), corrects the control actions of the automaton (position $P$ in Fig. 3, a) if he "does not agree" with the machine. If the human 
operator does not create corrective actions on the control body $\left(u_{c}=0\right)$, then the object is controlled only by automaton $\left(u=u_{a}\right)$. When the automaton shuts down or fails, the control of the object of the ergatic system is not interrupted, since it is continued by the human operator acting on the control body of the joint control apparatus [11].

\subsection{Simulation of object responses to discrete controls}

Programming the controlled movement of an object is performed using the hypothesis of complete certainty. The model of actions and responses of the human operator and the machine is used. In accordance with this model, the response of the object to the movement of the control bodies is determined by the set [6]

$$
Z=\left\{\dot{X}=F^{r}(X), r=1,2, \ldots, R\right\}
$$

of incomplete representations

$$
\dot{X}=F^{r}(X)
$$

of elementary movements of the image point in the state space

$$
\mathbf{R}^{1+N}=\left\{t \times x_{1} \times x_{2} \times \ldots \times x_{N}\right\}
$$

where $X$ - the column vector of state parameters (phase coordinates) $x_{1}, x_{2}, \ldots, x_{N}, F^{r}(X)$ the vector function of $X, r$ - the ordinal number assigned to the vector function $F^{r}(X)$ in the set $Z$. The point above the letter indicates the operation of differentiation with respect to time $t$.

The programmed motion of an object over a finite time interval is determined by the sequence of incomplete representations of elementary movements (9) from the set (8). The task of building such a sequence is to find the representation of a finite series of natural numbers $K, K+1, \ldots, M$ into the set (8), otherwise, into the set of natural numbers $r$ from 1 to $R$. Formulations and solutions of such problems should be carried out as the movement executes with the advent of new goals [12]. In accordance with it, it can be assumed that in the extreme case, repeated programming of the controlled movement by the set (8) each time as the movement is executed can be carried out for the entire life cycle of the ergatic human-machine system. Suppose, for example, that the first stage of the controlled movement of an object is programmed through a certain representation of numbers $1,2, \ldots$, 5 to the set (8) to achieve its goal and is implemented in accordance with the program, the second stage is programmed for a sequence of numbers $5,6, \ldots, 12$ and the new goal, the third stage is for the sequence $12,13, \ldots, K$, and so on. The control of the object at each subsequent stage of the program movement will be completely determined if a sequence of incomplete representations of elementary movements (9) is constructed for it, the time moments of the change of one elementary movement to another in this sequence are found, and the state of the object at the end of the previous stage is known. Thus, as the ergatic system is operated, the object control algorithms for each stage will change, and at each stage, the programmed motion of the object will be characterized by the movement of the image point along the trajectory in the state space (10). Each control algorithm subsequent after the completed stage of the controlled movement of the object, which has the form of a given sequence of incomplete representations of elementary movements (9), can be extracted from the "library" of algorithms or, if necessary, constructed as a result of interaction between the human operator and the control automaton through the humanmachine interface. Optimization of program control by the algorithm should lead to the uniqueness of the solution to the problem of determining the time moments of the change of elementary movements of an object in accordance with their incomplete representations at the stage of controlled movement. Necessary calculations are assigned to the computer of 
the control automaton interacting with the human operator. In this regard, we will consider the possibility of using mathematical modeling to perform the necessary calculations.

The joint control act (1) corresponding to any of the conditions (2), (3) or (4) - (7) is implemented by the human operator and the control automaton of the ergatic system as a structural control $[10,13]$, which on the set (8) of incomplete representations of motion (9) is a discrete control and is characterized by a triple of variables $\left\{t_{s}, F^{r}(X), F^{p}(X)\right\}$ under the conditions of $p \neq r, F^{p}(X) \neq F^{r}(X)$ [13]. Discrete control

$$
W_{r}^{p}\left(t_{s}\right)=\left\{t_{s}, F^{r}(X), F^{p}(X)\right\}
$$

expressed in the fact that at the moment of time $t_{s} \quad\left(t_{s}>t_{s-1}\right)$, the incomplete representation of motion (9) from the set $Z(8) \dot{X}\left(t_{s}-0\right)=F^{r}\left(X\left(t_{s}-0\right)\right)$ changes to the incomplete representation $\dot{X}\left(t_{s}+0\right)=F^{p}\left(X\left(t_{s}+0\right)\right)$ from the same set. Corresponding virtual discrete control signal

$$
\nabla(s)=F^{r}\left(X\left(t_{s}-0\right)\right)-F^{p}\left(X\left(t_{s}+0\right)\right)
$$

characterizes an abrupt change in the speeds of some (or all) phase coordinates, which is associated with a new local control goal and a transition to another representation of motion. The virtual discrete signal is expressed by a vector function $\nabla(s)$ of dimension $N \times 1$. A physically virtual discrete control signal corresponds, for example, the closure of contact 3 of the device in Fig. 2, a when the control body is moved from the position $Q$ to the position $P$ (model (2), (3) 2P), or the moment of an abrupt change in the speed of increase of the signal $v$ of the apparatus sensor in Fig. 3, a (model (4), (5) MP ), or other reactions of the joint control apparatus, modeled by movements of the control body (2) (7).

The use of models of discrete control signals (12) makes it possible to solve programming, reprogramming, and control problems for a wide range of human-machine interface devices on a unified methodological basis, including the devices sowed in Fig. 2,a and Fig. 3,a. In particular, this is possible when technical means of touch (contact) control are used on the basis of capacitive or resistive sensors, touch screens, alphanumeric and functional keys, and other devices. The justification for this possibility is caused by the fact that, using similar technical means, virtual discrete control signals (12) are generated not by non-switching contacts, as, for example, in the apparatus in Fig. 2, a, but by electronic keys [7] that implement structural control in the ergatic system. To display the intentions of the control automaton of the ergatic system, visual, sound and tactile signals can be applied [14].

It follows from the principle of constructing the a posteriori-formed control that the automatons of program and corrective controls interact with each other to exchange information and have a common output to the joint control apparatus (JCA in Fig. 1). In technical terms, these automatons can not be separated, and all the calculations necessary to control the object can be done using a single control computer.

\section{Discussion of the results}

Joint control of the object through the control bodies of the human-machine interface of the ergatic system allows, depending on the conditions of the actual movement of the object, combining the control functions of the human operator and the control automaton on an optimization basis. To increase the efficiency of joint control, it is proposed to use combined control apparatuses in which the manual drive for the control body is supplemented by an electric drive controlled by discrete signals from the control automaton 
of the system. The proposed options for constructing such devices provide for priority of a human operator over the automaton in controlling the object. There are three possibilities for implementing the controlled movement of the object when the goal of movement is achieved at a certain stage: program control according to the a priori description of the trajectory of movement in the form of a sequence of elementary movements with local goals; corrective control with the a posteriori-formed trajectory of motion, built on the principle of feedback, preserving the a priori sequence of elementary movements; reprogrammed control inside the selected target stage of the movement, implemented on an altered sequence of elementary movements as a variant of situational control.

The stationarity conditions presented in the study can be used for iterative calculations, for example, using the gradient method of optimal discrete controls for each of the implementations of controlled motion at a specific target stage in the life cycle of the ergatic system. Theoretical provisions allow using the mathematical apparatus of theories of ordinary differential equations and optimal control for the formulation and solution of problems of joint control of an object with lumped parameters by means of a control computer using numerical methods [15]. Thus, the advantages of automation in terms of accuracy and speed are realized in comparison with the capabilities of the human operator. At the same time, the possibilities to implement the control functions of a qualified human operator in complex and unforeseen situations, and to supplement them with the necessary calculations and programmable control performed by the control automaton are remained.

\section{Conclusion}

The practical application of joint control is advisable in ergatic human-machine systems, which are complex multi-purpose and multi-mode systems operating in a changing environment, especially in the a priori unpredictable variety, which is typical, for example, for moving objects in various environments. For example, the use of joint control in the "navigator-ship" system got positive results when solving problems of optimal speed and safe execution of the ship's entry into the lock chamber [6].

\section{References}

1. A.V. Vorobyov, M.M. Silvestrov, Y.I.Begichev, L.O. Kotitsyn., D.N. Levin, Mechatronics, automation, control 20(1), 59-64 (2019). DOI: 10.17587/mau.20.59-64

2. D.Y. Muromtsev, A.N. Gribkov, V.N. Shamkin, Informatics and control systems 3(53), 109118 (2017). DOI: $\underline{10.22250 / \text { isu.2016.53.109-118 }}$

3. A.B. Filimonov, N.B. Filimonov, Mechatronics, automation, control 19(9), 563-578 (2018). DOI: $10.17587 / \mathrm{mau} .19 .563-578$

4. A.V. Gulay, V.M. Zaitsev, Mechatronics, automation, control 20(10), 600-608 (2019). ID: 41143808

5. G. Stephanopoulos, C. Han, Computers \& Chemical Engineering 20(6-7), 743-791 (1996)

6. V.O. Tyrva, Bulletin of the Admiral Makarov State University of maritime and inland shipping 10(2), 439 - 443 (2018). DOI: 10.21821/2309-5180-2018-10-2-430-443

7. V.O. Tyrva, A.V. Saushev, O.V. Shergina, 2018 International Russian Automation Conference, RusAutoCon 2018, 8501665, DOI: 10.1109/RUSAUTOCON 2018.

8. Joint control (control by means of ACS). Aviation - commercial, civil, special aviation. [Electronic resource] - Access mode: http://ooobskspetsavia.ru/2015/11/16/sovmeshhennoeupravlenie-upravlenie-cherez-sau/ (reference date: 27.02.2018). 
9. Why is the Su-35 the most dangerous fighter in the world? [Electronic resource] - Access mode: http://www.kramola.info/vesti/novosti/pochemu-su-35-samyy-opasnyy-istrebitel-v-mire (reference date: 23.08.2017).

10.S.F. Sergeev, Mechatronics, automation, control 19(12), $806-811$ (2018). DOI: $\underline{10.17587 / \mathrm{mau} .19 .806-811}$

11.Maja Matarić, Dave Cliff,_Robotics and Autonomous Systems 19(1), 67-83 (1996).

12.Zhong-Jun Zhang, Jian-Bo Yang, Dong-Ling Xu. IFAC Proceedings Volumes 22(12), 13-18 (1989).

13. André da Motta Salles Barreto, Charles W. Anderson, Artificial Intelligence 172(4-5), 454-482 (2008).

14.Lee-Wen Tyan, Venkat Devarajan, Computer-Aided Design 30(5), 357-366 (1998).

15.A.B. Filimonov, N.B. Filimonov, A Optoelectronics, Instrumentation and Data Processing 53(4), 337-344 (2017). 